\title{
ON CROSSED PRODUCTS AND TAKAI DUALITY
}

\author{
by IAIN RAEBURN*
}

(Received 12th December 1986)

The Takai duality theorem has proved to be a fundamental tool in the theory of crossed products of $C^{*}$-algebras. It was inspired by Takesaki's duality theorem for crossed products of von Neumann algebras [7], so it is not surprising that the original proof [6] depended heavily on spatial techniques. Here we shall prove Takai's theorem by exploiting the universal properties of crossed products.

Let $\alpha: G \rightarrow$ Aut $A$ be an action of a locally compact group $G$ on a $C^{*}$-algebra $A$. We shall think of the crossed product as a $C^{*}$-algebra $A \times{ }_{\alpha} G$ whose representations are in one-to-one correspondence with the covariant representations of $(A, G, \alpha)$-that is, pairs of representations $\pi$ of $A, U$ of $G$ satisfying

$$
\pi\left(\alpha_{s}(a)\right)=U_{s} \pi(a) U_{s}^{*} \quad \text { for } a \in A, s \in G
$$

Of course, this has always been a standard way of viewing crossed products (see [2] and [1]), but as far as we know, no-one has bothered to give a precise characterisation in terms of this universal property. We therefore begin by giving a detailed definition of crossed product. It is easy to see that there is at most one $C^{*}$-algebra with the required property, and the usual construction of $A \times{ }_{a} G$ as the $C^{*}$-enveloping algebra of a Banach *-algebra $L^{1}(G, A)$ (as in $[4, \S 7.6]$, for example) shows that one exists. However, it is also easy to construct one directly, and we do this in Proposition 3.

When the group $G$ is abelian, there is a canonical action $\hat{\alpha}$ of the dual group $\hat{G}$ on $A \times{ }_{a} G$ (see Proposition 5), and Takai's duality theorem asserts that $\left(A \times{ }_{a} G\right) \times{ }_{a} \hat{G}$ is isomorphic to the tensor product $A \otimes \Re$ of $A$ with the algebra $\Re$ of compact operators on $L^{2}(G)$. The representations of $A \times G \times G$ are given by triples of representations $\pi$ of $A, U$ of $G, V$ of $\hat{G}$ on the same space; the main step in our proof is the construction of a representation of $A \otimes \Omega$ from such a triple, from which it will follow that $A \otimes \Omega$ has the universal property which characterises $A \times G \times \hat{G}$.

We feel that this proof is conceptually clearer than the usual one, and makes it easier to see what happens to additional structure under the duality isomorphism (see the remarks at the end of $\$ 2$ ). It is also technically quite elementary: we use only standard properties of multiplier algebras, as given in $[4, \S 3.12]$, some basic facts about

*This research was supported by the Australian Research Grants Scheme. 
integrating continuous functions of compact support with values in a $C^{*}$-algebra, and the fact that $C_{0}(G) \times G \cong \Omega\left(L^{2}(G)\right)$. The necessary integration theory is similar to that required in Pedersen's treatment [4], and we shall follow his example in relegating the details to an appendix. We discuss the isomorphism $C_{0}(G) \times G \cong \Omega$ in Example 4; however, we point out that it has also been a crucial ingredient in the previous versions.

We were led to this proof of Takai duality by attempts to make non-abelian duality work for (unreduced) crossed products by non-amenable groups, where there are severe problems with the usual spatial techniques. We are optimistic that one can prove, for example, a version of Quigg's duality theorem [5] along lines similar to these. However, this will involve considerable technical complications, and we hope that presenting the abelian case separately will illustrate the simplicity of the construction.

Notation. All homomorphisms and representations of a $C^{*}$-algebra $A$ will be *preserving. When we take tensor products of $C^{*}$-algebras, at least one will be nuclear, and we write $A \otimes B$ for the unique $C^{*}$-completion. If $\pi, v$ are commuting representations of $A, B$ on the same space $H$, we write $\pi \otimes v$ for the corresponding representation of $A \otimes B$ on $H$. The identity map will be denoted by $i$, and the identity of an algebra by 1 . We denote by $\mu$ a left Haar measure for a locally compact group $G$, by $\lambda, \rho$ the left and right regular representations of $G$ on $L^{2}(G)$, by $M$ the representation of $C_{0}(G)$ as multiplication operators on $L^{2}(G)$, and by $\tau, \sigma$ the actions of $G$ on $C_{0}(G)$ by left and right translation.

Let $M(B)$ denote the multiplier algebra of a $C^{*}$-algebra $B$. We shall call a homomorphism $\phi: A \rightarrow M(B)$ non-degenerate if there is an approximate identity $\left\{e_{i}\right\}$ for $A$ such that $\phi\left(e_{i}\right) \rightarrow 1$ strictly in $M(B)$; this implies that $\phi$ has a (unique) strictly continuous extension $\phi M(A) \rightarrow M(B)$ (see, for example, [3, Lemma 1.1]). When $B=\boldsymbol{\Omega}(H)$, so $M(B)=$ $B(H)$, such homomorphisms are also non-degenerate representations in the usual sense.

\section{Crossed products}

Let $\alpha: G \rightarrow$ Aut $A$ be a strongly continuous action of a locally compact group $G$ on a $C^{*}$-algebra $A$; we shall say $(A, G, \alpha)$ is a dynamical system. A covariant representation of $(A, G, \alpha)$ is a pair $(\pi, U)$ consisting of a nondegenerate representation $\pi$ of $A$ on $H$, and a (strongly continuous) unitary representation $U$ of $G$ on $H$, such that

$$
\pi\left(\alpha_{s}(a)\right)=U_{s} \pi(a) U_{s}^{*} \text { for } a \in A, s \in G .
$$

Definition 1. A crossed product for a dynamical system $(A, G, \alpha)$ is a $C^{*}$-algebra $B$ together with a homomorphism $i_{A}: A \rightarrow M(B)$ and a strictly continuous homomorphism $i_{G}: G \rightarrow U M(B)$ satisfying

(a) $i_{A}\left(\alpha_{s}(a)\right)=i_{G}(s) i_{A}(a) i_{G}(s)^{*}$ for $a \in A, s \in G$;

(b) for every covariant representation $(\pi, U)$ of $(A, G, \alpha)$, there is a non-degenerate representation $\pi \times U$ of $B$ with $\pi=(\pi \times U) \circ i_{A}$ and $U=(\pi \times U) \circ i_{G}$;

(c) the span of $\left\{i_{A}(a) i_{G}(z): a \in A, z \in C_{C}(G)\right\}$ is dense in $B$, where $i_{G}$ has been extended to $C_{C}(G)$ as in Corollary 8 . 
Remarks. (1) We shall shortly prove that there is a crossed product and that it is unique up to isomorphism. We shall therefore refer to the crossed product, and denote it by $A \times{ }_{\alpha} G$, as usual.

(2) Every non-degenerate representation $\rho$ of $A \times{ }_{\alpha} G$ has the form $\pi \times U$ for some covariant representation $(\pi, U)$ of $(A, G, \alpha)$. To see this, we take $\pi=\rho \circ i_{A}, U=\rho \circ i_{G}$, so that $(\pi, U)$ is covariant by (a). Lemma $7(3)$ implies that the equality $(\pi \times U) \circ i_{G}=U$ extends to $C_{C}(G)$; thus

$$
\pi \times U\left(i_{A}(a) i_{G}(z)\right)=\pi(a) U(z)=\rho\left(i_{A}(a) i_{G}(z)\right) \text { for } a \in A, z \in C_{C}(G),
$$

and it follows from (c) that $\rho=\pi \times U$.

(3) The homomorphisms $i_{A}$ and $i_{G}$ are necessarily injective. To see this, let $\pi$ be a faithful representation of $A$, and define a representation $\pi_{1}$ of $A$ on $L^{2}\left(G, H_{\pi}\right)$ by $\left(\pi_{1}(a) \xi\right)(s)=\pi\left(\alpha_{s}^{-1}(a)\right)(\xi(s))$. The pair consisting of $\pi_{1}$ and the action $1 \otimes \lambda$ of $G$ by left translation is covariant, so (b) implies that they factor through $i_{A}$ and $i_{G}$. But both $\pi_{1}$ and $1 \otimes \lambda$ are faithful, so $i_{A}$ and $i_{G}$ must be too.

Proposition 2. (1) There is a *homomorphism $i_{A} \times i_{G}$ of $C_{C}(G, A)$, with the *-algebra structure as in $[4, \S 3.6]$, onto a dense subalgebra of $A \times{ }_{\alpha} G$, such that $i_{A} \times i_{G}(a \otimes z)=$ $i_{A}(a) i_{G}(z)$, and

$$
\left\|i_{A} \times i_{G}(w)\right\| \leqq \mu(\operatorname{supp} w)\|w\|_{\infty} \quad \text { for } w \in C_{C}(G, A)
$$

(2) Let $\phi$ be a non-degenerate homomorphism of $A$ into the multiplier algebra $M(C)$ of a $C^{*}$-algebra $C$, and $u$ a strictly continuous homomorphism of $G$ into $U M(C)$ such that $\phi\left(\alpha_{s}(a)\right)=u_{s} \alpha(a) u_{s}^{*}$. Then there is a non-degenerate homomorphism $\phi \times u$ of $A \times{ }_{\alpha} G$ into $M(C)$ such that $(\phi \times u) \circ i_{A}=\phi$ and $(\phi \times u) \circ i_{G}=u$ on both $G$ and $C_{C}(G)$.

Proof. (1) Given $w \in C_{c}(G, A)$, we apply Lemma 7 to the strictly continuous map $s \rightarrow i_{A}(w(s)) i_{G}(s)$ to obtain an element

$$
i_{A} \times i_{G}(w)=\int i_{A}(w(s)) i_{G}(s) d s \in M(C),
$$

whose norm is bounded by $\mu(\operatorname{supp} w)\|w\|_{\infty}$. It is easy to check that $i_{A} \times i_{G}(a \otimes z)$ is as asserted, and approximating $w$ uniformly by a finite sum $\sum a_{i} \otimes z_{i}$ shows that in fact $i_{A} \times i_{G}(w)$ belongs to $A \times G$. It follows as in the proof of Corollary 8 that $i_{A} \times i_{G}$ is a ${ }_{-}$ homomorphism.

(2) We represent $C$ faithfully on a Hilbert space $H$, so that $(\phi, u)$ becomes a covariant representation, and apply property (b) to obtain a map of $A$ into $B(H)$ satisfying $(\phi \times u) \circ i_{A}=\phi$ and $(\phi \times u) \circ i_{G}=u$. An application of Lemma 7(3) shows that the second equation extends to $C_{C}(G)$; thus $\phi \times u$ carries the generator $i_{A}(a) i_{G}(z)$ into $\phi(a) u(z)$, and hence maps $A$ into $M(C) \subset B(H)$ (this also shows that $\phi \times u$ is independent of the representation chosen). If $\left\{z_{j}\right\} \subset C_{C}(G)$ is an approximate identity for the inductive limit topology, then it is easy to verify that $u\left(z_{j}\right) \rightarrow 1$ strictly in $M(C)$, and the nondegeneracy of $\phi \times u$ follows from that of $\phi$. 
Proposition 3. (1) If $\left(B, i_{A}, i_{G}\right)$ and $\left(C, j_{A}, j_{G}\right)$ are both crossed products for $(A, G, \alpha)$, there is an isomorphism $\phi$ of $B$ onto $C$ such that $\phi \circ i_{A}=j_{A}$ and $\phi \circ i_{G}=j_{G}$.

(2) There is a crossed product for any dynamical system.

Proof. (1) It follows from (c) and its adjoint that $j_{A}$ is non-degenerate, so by Proposition 2(2) there is a non-degenerate homomorphism $\phi=j_{A} \times j_{G}$ of $B$ into $M(C)$ such that $\phi\left(i_{A}(a) i_{G}(z)\right)=j_{A}(a) j_{G}(z)$. Condition (c) thus implies that $\phi$ maps $B$ onto $C$. Reversing the roles of $B$ and $C$ gives a homomorphism which also swaps generators, and hence is an inverse for $\phi$.

(2) Choose a set $S$ of covariant representations of $(A, G, \alpha)$ such that every cyclic covariant representation is equivalent to a member of $S$. (To see that there is such a set, fix a Hilbert space of large cardinality, and restrict attention to representations in this space.) We then let $H=\oplus\left\{H_{\pi}:(\pi, U) \in S\right\}$, define $i_{A}=\oplus \pi: A \rightarrow B(H), i_{G}=\oplus U$, and take $B$ to be the closed span of $\left\{i_{A}(a) i_{G}(z): a \in A, z \in C_{C}(G)\right\}$. To prove that $B$ is a $C^{*}$-algebra, we first note that if $f \in C_{C}(G, A)$, then Lemma 7 gives us an operator

$$
i_{A} \times i_{G}(f)=\int i_{A}(f(s)) i_{G}(s) d s \in B(H)=M(\Omega(H)) ;
$$

by approximating $f$ uniformly by $\sum a_{i} \otimes z_{i} \in A \otimes C_{C}(G)$ and using Lemma 7(1) we can see that $i_{A} \times i_{G}(f) \in B$. Now $\left(i_{A}, i_{G}\right)$ is covariant, so it follows that

$$
\left(i_{G}(z) i_{A}(a)\right)=\left(\int z(s) i_{G}(s) d s\right) i_{A}(a)=\int z(s) i_{G}(s) i_{A}(a) d s=\int z(s) i_{A}\left(\alpha_{s}(a)\right) i_{G}(s) d s
$$

belongs to $B$. Since $i_{G}(z)^{*}=i_{G}\left(z^{*}\right)$, this implies that $B$ is closed under taking adjoints. Further, since we can approximate $i_{G}(z) i_{A}(b)$ by a finite sum $\sum i_{A}\left(b_{i}\right) i_{G}\left(z_{i}\right)$, we can approximate $i_{A}(a) i_{G}(z) i_{A}(b) i_{G}(w)$ by a sum

$$
\sum i_{A}(a) i_{A}\left(a_{i}\right) i_{G}\left(z_{i}\right) i_{G}(w)=\sum i_{A}\left(a a_{i}\right) i_{G}\left(z_{i} \times w\right),
$$

and $B$ is also closed under multiplication, hence a $C^{*}$-algebra. The same argument shows that $i_{A}$ takes values in $M(B)$. We also have

$$
i_{G}(s) i_{A}(a) i_{G}(z)=\int i_{A}\left(\alpha_{s}(a)\right) z(t) i_{G}(s t) d t,
$$

and a similar formula for $i_{A}(a) i_{G}(z) i_{G}(s)$, so $i_{G}(s)$ also belongs to $M(B)$. Property (a) follows from the covariance of $\left(i_{A}, i_{G}\right)$ and (c) is trivially true. For $(\pi, U) \in S$, we can define $\pi \times U$ by projecting onto the subspace $H_{n}$ of $H$, and we can handle an arbitrary covariant representation by decomposing it as a direct sum of cyclic representations and conjugating appropriate members of $S$. Thus (b) also holds, and $B$ is a crossed product for $(A, G, \alpha)$.

Example 4. We shall later need to know that $\left(\Omega\left(L^{2}(G)\right), M, \lambda\right)$ is a crossed product for $\left(C_{0}(G), G, \tau\right)$, and we now briefly indicate hov' this can be proved. The covariance of $(M, \lambda)$ gives (a). The integrated form $M \times \lambda$ (see Proposition 2 above) carries $C_{C}\left(G, C_{c}(G)\right)$ into the space of operators given by kernels in $C_{C}(G \times G)$; these are dense 
in $\Re\left(L^{2}(G)\right.$ ), and (c) follows by a standard approximation argument. The imprimitivity theorem implies that every covariant representation of $\left(C_{0}(G), G, \tau\right)$ is induced from the trivial subgroup $\{e\}$. The representations of $\{e\}$ are just the multiples of the trivial one, which induces to $(M, \lambda)$, so every covariant representation of $\left(C_{0}(G), G\right)$ is equivalent to a multiple of $(M, \lambda)$. This gives (b).

Proposition 5. Let $(A, G, \alpha)$ be a dynamical system, with $G$ abelian. Then there is an action $\hat{\alpha}$ (the dual action) of $\hat{G}$ on $A \times{ }_{\alpha} G$ such that

$$
\hat{\alpha}_{\gamma}\left(i_{A}(a) i_{G}(z)\right)=i_{A}(a) i_{G}(\gamma z) \text { for } \gamma \in \hat{G}, a \in A, z \in C_{C}(G) .
$$

Proof. Define $j_{\gamma}: G \rightarrow U M(B)$ by $j_{\gamma}(s)=\gamma(s) i_{G}(s)$. We claim that the triple $\left(B, i_{A}, j_{\gamma}\right)$ is also a crossed product for $(A, G, \alpha)$. In fact (c) holds because $j_{\gamma}(z)=i_{G}(\gamma z)$, and both (a) and (b) follow from the observation that $(\pi, U)$ is a covariant representation iff $(\pi, \gamma U)$ is. Thus by part (1) of Proposition 3, there is an isomorphism $\hat{Q}_{\gamma}: B \rightarrow B$ such that

$$
\hat{\alpha}_{y}\left(i_{A}(a) i_{G}(z)\right)=i_{A}(a) j_{y}(z)=i_{A}(a) i_{G}(\gamma z) .
$$

The second formula for $\hat{\alpha}_{\gamma}$ implies that $\gamma \rightarrow \hat{\alpha}_{\gamma}$ is a homomorphism, and from Proposition 2 we have

$$
\begin{aligned}
\left\|i_{A}(a) i_{G}(\gamma z)-i_{A}(a) i_{G}(z)\right\| & =\left\|i_{A} \times i_{G}(a \otimes \gamma z-a \otimes z)\right\| \\
& \leqq \mu(\operatorname{supp} z)\|a\|\|\gamma z-z\| \\
& \rightarrow 0 \text { as } \quad \gamma \rightarrow 1,
\end{aligned}
$$

so $\gamma \rightarrow \hat{\alpha}_{y}$ is continuous.

\section{The Takai duality theorem}

Theorem 6. Let $(A, G, \alpha)$ be a dynamical system with $G$ abelian. There is an isomorphism $\phi$ of $\left(A \times{ }_{\alpha} G\right) \times{ }_{\alpha} \hat{G}$ onto $A \otimes \mathfrak{A}\left(L^{2}(G)\right)$ such that the second dual action ${ }^{\hat{\alpha}}$ of $G=(\hat{G})^{\wedge}$ is carried into $\alpha \otimes(\operatorname{Ad} \rho)$.

Proof. Let $\alpha, \alpha^{-1}$ denote the maps of $A$ into $C_{b}(G, A) \subset M\left(C_{0}(G, A)\right)$ given by

$$
a(a)(s)=\alpha_{s}(a), \quad \alpha^{-1}(a)(s)=\alpha_{s}^{-1}(a),
$$

and define embeddings of $A, G$ and $\hat{G}$ in $M(A \otimes \Omega)$ by

$$
j_{A}=(i \otimes M) \circ \alpha^{-1}, \quad j_{G}=1 \otimes \lambda, \quad j_{G}=1 \otimes M,
$$

where we are viewing $\hat{G}$ as a subset of $C_{b}(G)$. If $\left\{e_{i}\right\}$ is an approximate identity for $A$, then routine arguments show that $\alpha\left(e_{i}\right) \rightarrow 1$ strictly in $M\left(A \otimes C_{0}(G)\right)$, and hence $j_{A}\left(e_{i}\right) \rightarrow 1$ strictly in $M(A \otimes \Re)$. Further, we have 


$$
\begin{aligned}
j_{A}\left(\alpha_{s}(a)\right) & =i \otimes M\left(\alpha^{-1}\left(\alpha_{s}(a)\right)\right)=i \otimes M\left(i \otimes \tau_{s}\left(\alpha^{-1}(a)\right)\right) \\
& =\left[i \otimes\left(\left(\operatorname{Ad} \lambda_{s}\right) \circ M\right)\right]\left(\alpha^{-1}(a)\right)=j_{G}(s) j_{A}(a) j_{G}(s)^{*} .
\end{aligned}
$$

Thus by Proposition 2(2), there is a non-degenerate homomorphism $j_{A} \times j_{G}$ of $A \times{ }_{\alpha} G$ into $M(A \otimes R)$, such that $\left(j_{A} \times j_{G}\right) \circ i_{A}=j_{A}$ and $\left(j_{A} \times j_{G}\right) \circ i_{G}=j_{G}$ on both $G$ and $C_{C}(G)$. We shall prove that $\left(A \otimes \mathcal{R}, j_{A} \times j_{G}, j_{G}\right)$ is a crossed product for $\left(A \times{ }_{\alpha} G, G, \hat{\alpha}\right)$, and the result will then follow easily.

The commutativity of $C_{b}(G)$ implies that $j_{A}(a)$ commutes with $j_{G}(\gamma)$, and it is easy to check that $M(\gamma) \lambda(z)=\lambda(\gamma z) M(\gamma)$, so

$$
j_{G}(\gamma) j_{A}(a) j_{G}(z) j_{G}(\gamma)^{*}=j_{A}(a) j_{G}(\gamma z)=\hat{\alpha}_{y}\left(j_{A}(a) j_{G}(z)\right),
$$

which implies condition (a) of Definition 1. Next we note that, if $w \in C_{C}(\hat{G})$ and $F^{-1}$ denotes the inverse of the usual Fourier transform, then $j_{G}(w)=1 \otimes M\left(F^{-1} w\right)$. The range of $F^{-1}$ is a dense subalgebra of $C_{0}(G)$, so we can use standard approximation arguments to see that the span of $\left\{\alpha^{-1}(a)\left(1 \otimes\left(F^{-1} w\right)\right)\right\}$ is dense in $A \otimes C_{0}(G)$. This and the density of $\{M(f) \lambda(z)\}$ in $\Omega\left(L^{2}(G)\right)$ (see Example 4 above) imply that the set

$$
\left\{j_{A}(a) j_{G}(z) j_{G}(w): a \in A, z \in C_{C}(G), w \in C_{C}(\hat{G})\right\}
$$

spans a dense subspace of $A \otimes \Re$. Since $j_{A} \times j_{G}\left(i_{A}(a) i_{G}(z)\right)=j_{A}(a) j_{G}(z)$, this shows that condition (c) also holds, and it remains to verify (b).

Let $(\pi \times U, V)$ be a covariant representation of $(A \times G, \hat{G}, \hat{\alpha})$. We shall construct the corresponding representation of $A \otimes \Re$ by tensoring commuting representations of $A$ and $\boldsymbol{\Omega}$. Composing the integrated form of $V$ with the Fourier transform $F$ gives a representation $L$ of $C_{0}(G)$, whose extension to $C_{b}(G)=M\left(C_{0}(G)\right)$ satisfies $L(\gamma)=V_{\gamma}$. The equation relating $U$ and $V$ implies that $(L, U)$ is a covariant representation of $\left(C_{0}(G), G, \tau\right)$, and hence there is a representation $L \times U$ of $\mathcal{R}\left(L^{2}(G)\right)$ such that $(L \times U) \circ M=L$ and $(L \times U) \circ \lambda=U$ (see Example 4). Since each $V_{y}$ commutes with the range of $\pi$, so does each $L(f)$, and they combine to give a representation $\pi \otimes L$ of $A \otimes C_{0}(G)$. Let $\rho$ denote the representation $(\pi \otimes L) \circ \alpha$ of $A$ on $H$. It is immediate that $\rho$ commutes with $L$, and we claim it commutes with $U$ too.

Suppose $\left\{\phi_{n}\right\} \subset A \odot C_{0}(G)$ converges to $\alpha(a)$ uniformly on compacta, so that $\pi \otimes L(\alpha(a))$ is the *-strong limit of $\pi \otimes L\left(\phi_{n}\right)$. Then for fixed $s \in G$ we also have

$$
\alpha_{s} \otimes \tau_{s}\left(\phi_{n}\right)(t)=\alpha_{s}\left(\phi_{n}\left(s^{-1} t\right)\right) \rightarrow \alpha_{s}\left(\alpha(a)\left(s^{-1} t\right)\right)=\alpha_{t}(a)=\alpha(a)(t)
$$

uniformly on compacta in $G$. Thus, given $\xi \in H$, we can choose $\phi=\sum a_{i} \otimes f_{i} \in A \odot C_{0}(G)$ such that

$$
\pi \otimes L(\phi) \xi \sim \pi \otimes L(\alpha(a)) \xi \quad \text { and } \quad \pi \otimes L\left(\alpha_{s} \otimes \tau_{s}(\phi)\right) U_{s} \xi \sim \pi \otimes L(\alpha(a)) U_{s} \xi .
$$

Then

$$
U_{s}(\pi \otimes L(\alpha(a)) \xi) \sim U_{s}\left(\sum \pi\left(a_{i}\right) L\left(f_{i}\right) \xi\right)
$$




$$
\begin{aligned}
& =\sum \pi\left(\alpha_{s}\left(a_{i}\right)\right) U_{s} L\left(f_{i}\right)(\xi) \\
& =\sum \pi\left(\alpha_{s}\left(a_{i}\right)\right) L\left(\tau_{s}\left(f_{i}\right)\right)\left(U_{s} \xi\right) \\
& \sim \pi \otimes L(\alpha(a))\left(U_{s} \xi\right) .
\end{aligned}
$$

This approximation can be made arbitrarily accurate, so we have proved that $U_{s} \rho(a)=$ $\rho(a) U_{s}$.

Since both $L$ and $U$ commute with $\rho$, so does $L \times U$, and we obtain a representation $v=\rho \otimes(L \times U)$ of $A \otimes \Re$ on $H$. At once we have

$$
\begin{gathered}
\nu \circ j_{G}(\gamma)=(\rho \otimes(L \times U))(1 \otimes M(\gamma))=(L \times U)(M(\gamma))=L(\gamma)=V_{\gamma}, \\
v \circ j_{G}(s)=(\rho \otimes(L \times U))\left(1 \otimes \lambda_{s}\right)=(L \times U)\left(\lambda_{s}\right)=U_{s} .
\end{gathered}
$$

Next we observe that $\alpha$ extends to an automorphism $\beta$ of $A \otimes C_{0}(G)$ satisfying $\beta(a \otimes f)=$ $\alpha(a)(1 \otimes f)$ : when realised on $C_{0}(G, A), \beta$ is given by $\beta(z)(s)=\alpha_{s}(z(s))$. A quick calculation shows

$$
[((\pi \otimes L) \circ \alpha) \otimes(L \times U)] \circ(i \otimes M)=(\pi \otimes L) \circ \beta,
$$

so that

$$
\begin{aligned}
\nu \circ j_{A}(a) & =[((\pi \otimes L) \circ \alpha) \otimes(L \times U)] \circ(i \otimes M) \circ \alpha^{-1}(a) \\
& =(\pi \otimes L) \circ \beta \circ \alpha^{-1}(a) \\
& =(\pi \otimes L)(a \otimes 1)=\pi(a) .
\end{aligned}
$$

Thus $v \circ\left(j_{A} \times j_{G}\right) \circ i_{A}=v \circ j_{A}=\pi, \quad v \circ\left(j_{A} \times j_{G}\right) \circ i_{G}=U$, and $v \circ\left(j_{A} \times j_{G}\right)=\pi \times U$. This completes the proof of $(\mathbf{b})$.

Let $i_{1}$ denote the embedding of $A \times G$ in $M((A \times G) \times \hat{G})$. Then Proposition 3(1) gives us an isomorphism $\psi: A \otimes \Re \rightarrow A \times G \times \hat{G}$ such that

$$
\psi\left(j_{A}(a) j_{G}(z) j_{G}(w)\right)=\psi\left(j_{A} \times j_{G}\left(i_{A}(a) i_{G}(z)\right) j_{G}(w)\right)=i_{1}\left(i_{A}(a) i_{G}(z)\right) i_{G}(w)
$$

Now $\hat{\alpha}_{s}$ carries the generator on the right of $(1)$ to $i_{1}\left(i_{A}(a) i_{G}(z)\right) i_{G}(s w)$, so to verify that $\psi^{-1} \circ \hat{\alpha} \circ \psi=\alpha \otimes(\operatorname{Ad} \rho)$ we need only check that

$$
\alpha_{s} \otimes\left(\operatorname{Ad} \rho_{s}\right)\left(j_{A}(a) j_{G}(z) j_{G}(w)\right)=j_{A}(a) j_{G}(z) j_{G}(s w) .
$$

A quick calculation gives $\operatorname{Ad} \rho_{s}(M(f))=M\left(\sigma_{s}(f)\right)$, from which we obtain

$$
j_{G}(s w)=1 \otimes M\left(F^{-1}(s w)\right)=1 \otimes M\left(\sigma_{s}\left(F^{-1} w\right)\right)=1 \otimes\left(\operatorname{Ad} \rho_{s}\left(j_{G}(w)\right)\right) .
$$


We also have

$$
\alpha_{s} \otimes\left(\operatorname{Ad} \rho_{s}\right)\left(j_{A}(a)\right)=i \otimes M\left(\alpha_{s} \otimes \sigma_{s}\left(\alpha^{-1}(a)\right)\right)=i \otimes M\left(\alpha^{-1}(a)\right)=j_{A}(a),
$$

and $\rho_{s}$ commutes with $\lambda(z)$, which gives (2). This completes the proof of the theorem.

Remark. In the original proof of this theorem, the isomorphism $\psi$ is constructed in three stages, and it can be quite painful to extract additional information about $\psi$. This should usually be easier to do using our version. For example, (1) describes what $\psi$ does to a set of generators for $A \otimes \Re$, and our construction shows that, for any representation $(\pi \times U) \times V$ of $A \times G \times \hat{G}$, we have

$$
((\pi \times U) \times V) \circ \psi^{-1}=((\pi \otimes L) \circ \alpha) \otimes(L \times U),
$$

where $L$ is the representation of $C_{0}(G)$ corresponding to the representation $V$ of $\hat{G}$. For another example, suppose an abelian $C^{*}$-algebra $C$ acts on $A$ (so that we have a map of $C$ into the centre of $M(A)$ ), and that each automorphism $\alpha_{s}$ commutes with this action. Then there are natural actions of $C$ on $A \times{ }_{\alpha} G,\left(A \times{ }_{\alpha} G\right) \times{ }_{\alpha} \hat{G}$ and $A \otimes R$, and it follows from (1) that $\psi$ is a $\mathrm{C}$-module isomorphism.

\section{Appendix on integration}

Lemma 7. Let $f \in C_{C}(G, A)$. Then there is a unique element $\int f(s) d s$ of $A$ such that for any nondegenerate representation $\pi$ of $A$

$$
\left(\pi\left(\int f(s) d s\right) \xi, \eta\right)=\int(\pi(f(s)) \xi, \eta) d s \quad \text { for } \xi, \eta \in H_{\pi} .
$$

Further, we have

(1) $\left\|\int f(s) d s\right\| \leqq \mu(\operatorname{supp} f)\|f\|_{\infty}$;

(2) $\left(\int f(s) d s\right) a=\int f(s) a d s \quad$ for $a \in A$ or $M(A)$;

(3) $\phi\left(\int f(s) d s\right)=\int \phi(f(s)) d s$ for any homomorphism $\phi: A \rightarrow B$,

(4) $\left(\int f(s) d s\right)^{*}=\int f(s)^{*} d s$.

Similarly, if $f: G \rightarrow M(A)$ is strictly continuous and has compact support, there is an element $\int f(s) d s$ of $M(A)$ satisfying $\left(^{*}\right),(1),(2),(4)$. If $\phi$ is non-degenerate, so that $\phi$ extends to $M(A)$, (3) also holds.

Proof. If $\pi$ is a faithful representation of $A$, the right-hand side of $\left(^{*}\right)$ is a bounded sesquilinear form on $H_{n}$, and hence defines a bounded operator $T(f)$ on $H_{\pi}$ satisfying

$$
\|T(f)\| \leqq \mu(\operatorname{supp} f)\|f\|_{\infty} .
$$

To see that $T(f)$ belongs to $\pi(A)$, we approximate $f$ uniformly on supp $f$ by a finite. 
sum $\sum a_{i} \otimes z_{i} \in A \otimes C_{C}(G)$, so that by (5), $T(f)$ is near $T\left(\sum a_{i} \otimes z_{i}\right)$. It is easy to check that

$$
T\left(\sum a_{i} \otimes z_{i}\right)=\sum \pi\left(a_{i}\right)\left(\int z_{i}(s) d s\right) \in \pi(A),
$$

so $T(f) \in \pi(A)$. We define $\int f(s) d s=\pi^{-1}(T(f))$. Notice that $\int f(s) d s$ is near

$$
\pi^{-1}\left(T\left(\sum \alpha_{i} \otimes z_{i}\right)\right)=\sum a_{i} \int z_{i}(s) d s,
$$

so for any representation $\rho$ of $A, \rho\left(\int f(s) d s\right)$ is close to $\sum \rho\left(a_{i}\right) \int z_{i}(s) d s$, and it follows that $\left(^{*}\right)$ holds. Since $\pi$ is isometric, (5) implies (1); (2), (3) and (4) follow from $\left({ }^{*}\right)$.

If $f: G \rightarrow M(A)$, the same argument gives an operator $T(f)$ on $H_{n}$, and we can see that it belongs to $M(A)$ by making uniform approximations to the functions $s \rightarrow f(s) a, s \rightarrow a f(s)$. Then (1), (2) and (4) are easy to check. By definition of $\phi$ on $M(A)$ (see [3, Lemma 1.1]), we have

$$
\begin{aligned}
\phi\left(\int f(s) d s\right)(\phi(a) b) & =\phi\left(\left(\int f(s) d s\right) a\right) b \\
& =\phi\left(\int f(s) a d s\right) b \quad \text { by (2) } \\
& =\left(\int \phi(f(s) a) d s\right) b \quad \text { by (3) } \\
& =\int \phi(f(s) a) b d s \\
& =\int \phi(f(s))(\phi(a) b) d s \\
& =\left(\int \phi(f(s)) d s\right)(\phi(a) b),
\end{aligned}
$$

which gives (3).

Corollary 8. Suppose $u$ is a strictly continuous homomorphism of $G$ into $U M(A)$. Then $u(z)=\int z(s) u_{s} d s$ defines a ${ }^{*}$-homomorphism of $C_{C}(G)$ into $M(A)$, which is continuous for the inductive limit topology on $C_{C}(G)$.

Proof. We apply the lemma to the strictly continuous map $s \rightarrow z(s) u_{s}$. To see that $u$ is a homomorphism on $C_{C}(G)$, we represent $A$ on a Hilbert space. Then by $\left({ }^{*}\right)$ and Fubini's theorem

$$
\begin{aligned}
(u(z \times w) \xi, \eta) & =\int\left\{\int z(t) w\left(t^{-1} s\right) d t\right\}\left(u_{s} \xi, \eta\right) d s \\
& =\int z(t)\left\{\int w(s)\left(u_{t} u_{s} \xi, \eta\right) d s\right\} d t \\
& =\int z(t)\left(u(w) \xi, u_{t}^{*} \eta\right) d t \\
& =(u(z) u(w) \xi, \eta) .
\end{aligned}
$$

A similar argument shows that $u\left(z^{*}\right)=u(z)^{*}$, and the continuity follows from (1). 


\section{REFERENCES}

1. S. Doplicher, D. Kastler and D. W. Robinson, Covariance algebras in field theory and statistical mechanics, Comm. Math. Phys. 3 (1966), 1-28.

2. J. Glimm, Families of induced representations, Pacific J. Math. 12 (1962), 885-911.

3. M. B. Landstad, J. Phillips, I. Raeburn and C. E. Sutherland, Representations of crossed products by coactions and principal bundles, Trans. Amer. Math. Soc. 299 (1987), 747-784.

4. G. K. Pedersen, $C^{*}$-algebras and their automorphism groups (Academic Press, London and New York, 1979).

5. J. C. QuigG, Duality for reduced twisted crossed products of $C^{*}$-algebras, Indiana Univ. Math. J. 35 (1986), 549-571.

6. H. TaKal, On a duality for crossed products of $C^{*}$-algebras, J. Funct. Analysis 19 (1975), 25-39.

7. M. TAKESAKI, Duality for crossed products and the structure of von Neumann algebras of type III, Acta. Math. 131 (1973), 249-310.

School of Mathematics

University of New South Wales

P.O. Box 1

KENSINGTON

NSW 2033

Australia 